\title{
Topical anesthesia in cosmetic dermatological procedures
}

Jacek Zdybski ${ }^{1}$, Ewa Trznadel-Grodzka²

${ }^{1}$ Dermatology Clinic, Ostrowiec Swietokrzyski, Poland, ${ }^{2}$ Department of Dermatology, Pediatric Dermatology and Oncology,

Medical University of Lodz, Lodz, Poland

Corresponding author: Dr. Jacek Zdybski, E-mail: jacek_z@icloud.com

\begin{abstract}
Background: Rapid increase in demand for fast, effective and painless cosmetic procedures has inspired the pursue for the ideal method of anesthesia. The aim of the study was to compare the effectiveness of different types of topical anesthetics most commonly utilized in dermatological procedures: EMLA cream, BLT cream, infraorbital nerve blocks with 2\% lidocaine with epinephrine. Material and Methods: The study involved 12 patients with scheduled painful dermatological procedures intended to improve their facial and neck skin. Pain intensity was measured using 0-10 numeric scale (10 being the worst imaginable pain and 0 no pain). Patients were asked to assess their pain levels during fractionated CO2 laser treatment of the face. Results: Results demonstrated that patients subjected to treatment with the use of anesthetic EMLA and BLT creams still feel a considerable level of pain during the performed dermocosmetic procedures. Similar pain level concerned the group anesthetized with the infraorbital nerve block injection. Conclusion: Finding the ideal topical anesthetic is one of the greatest challenges of present-day cosmetic dermatology. The most desired method of anesthesia in this context would have to be easy to apply, display high clinical effectiveness over a short time period, be able to exert numbing effect on intact skin without systemic effects, cause nominal pain or discomfort during treatment with minimal to no side effects. According to the results of the reported study, the most effective method of anesthesia was proved to be the triple anesthesia involving of a combination of a painkiller drug, EMLA cream and infraorbital nerve block.
\end{abstract}

Key words: Topical anesthesia; EMLA; BLT; Nerve block; Lidocaine; Cosmetic procedures

\section{INTRODUCTION}

In recent decades cosmetic dermatology has experienced an unprecedented growth in popularity. Rapid increase in demand for fast, effective and painless cosmetic procedures has simultaneously inspired the pursue for the ideal method of anesthesia. Currently, the field of cosmetic procedures is increasingly being dominated by non-surgical (but nevertheless invasive and potentially painful) procedures, often described as 'quick and safe' alternatives to surgery. Patients thus expect quick yet spectacular results achieved through procedures that can be safely carried out within a span of a lunch-break. To meet their demand, dermatologists are constantly searching for anesthetics that would be most effective but also yield the least risk for a patient and bear minimal (if any at all), unwanted side-effects.

\section{Anesthesia in Cosmetic Dermatology Practice}

There are many types of anesthesia that are employed by dermatologists during cosmetic procedures. Out of the broad range of available options, local or topical anesthesia has become the preferred method of practitioners. The popularity of topical anesthesia during dermocosmetic procedures is undoubtedly linked to the unique nature of these procedures and the fact that they are usually associated with precise medical interventions that are however usually limited to very small areas of skin. At the same time, as many such procedures are typically carried out on the patient's face, the associated pain and the fear of pain and/or needles is an important factor that should be addressed while deciding upon the best method of anesthesia. 
Effective pain management strategies adjusted to the type of procedure planned for a given patient is critical to achieving the desired effect of the performed procedure while assuring a high level of overall patient satisfaction. Pain is a common barrier to cosmetic procedures, and therefore alleviating the fear of painful interventions is critical factor determining the patient's assessment of the procedure, the skill of the medical staff and the procedure itself. The level of pain associated with the given type of procedure may also be a defining element in patient's decision concerning the continuation of therapy, the choice of the type of further therapy and evaluation of the medical center. It should be also always considered that patients checking in for dermocosmetic procedures are prevailingly driven by the desire to improve their appearance, undergo corrections of psychical beauty defects or to simply look younger. This specific group of patients is thus mostly concentrated on achieving a certain aesthetical outcome of the chosen medical procedure and as they are not suffering from a medical condition they are less inclined to accept pain as part of their therapy. In may regards patients deciding to undergo a cosmetic dermatological procedure are perceiving these procedures as an extended (or rather-advanced) form of beauty treatment and as such are expecting them to be fast, effective and painless.

These patients' expectations along with the dynamic increase in popularity of cosmetic dermatology determine the need to search for the best ways of eliminating unwanted side-effects and relieve the pain and the stress related with the discussed types of procedures.

Local anesthesia is therefore broadly regarded as the most effective and convenient component of successful interventions in cosmetic dermatology. Its possible administration techniques and types of medications are varied, allowing for selecting the most appropriate type of anesthesia given the nature of the procedure and the patient in question.

\section{Types of Topical Anesthetics}

Local anesthetics alleviate pain by the inhibition of voltage-gated sodium channels which in turn prevents the depolarization of nerve cells and reversibly blocs nerve conduction $[1,2]$. Their operation therefore causes temporary loss of sensation in a limited area of application.
Chemical structure of all topical anesthetics is uniform; they contain a lipophilic aromatic group, an intermediate chain, and a hydrophilic amine group. The chemical group attached to the intermediate chain determines their classification as amide- (eg, lidocaine, bupivacaine, articaine, mepivacaine, prilocaine, levobupivacaine) or ester-containing (eg, procaine, proparacaine, benzocaine, chlorprocaine, tetracaine, cocaine). Epinephrine or another adrenergic agonist is often added to the local topical agent in order to prolong the anesthetic effects by increasing vasoconstriction and decreasing the rate of systemic absorption [3]. The role of epinephrine in this context is also to reduce surgical bleeding and increase the intensity of blocade.

These topical anesthetics can be administered in a multitude of methods, including topical sprays, patches, creams, ointments, peels and even injections. Duration of action of various formulations depends on the agent and chosen method. Their distinctive feature is that they noninvasively deliver anesthesia in locally required areas.

There are several clinical techniques of delivering anesthesia locally:

1) Topical, non-invasive administration by means of applying the drug through spray, ointment or cream

2) Topical refrigerants such as the cooling ethyl chloride spray

3) Infiltration anesthesia with the use of drugs intended for topical anesthesia

4) Block anesthesia for example - peripheral nerve block (as in the case of this study) which is an injection of a topical anesthetic in the vicinity of a peripheral nerve to anesthetize that nerve's area of innervations

5) Anesthesia through infiltrative tumescent technique.

The most often used method among practicing dermatologists is the EMLA cream. EMLA stands for Eutectic Mixture of Local Anaesthetics and is a mixture of lidocaine $25 \mathrm{mg} / \mathrm{g}$, prilocaine $25 \mathrm{mg} / \mathrm{g}$, arlatone 289 (emulgent), carbapol 934 (thickener) and distilled water in $1 \mathrm{ml}$ cream.

EMLA cream has proven to have anesthetic efficacy in several clinical trials involving such dermatologic procedures as cryotherapy, pulsed dye laser treatment, and debridement of leg ulcers [4-7]. It is used worldwide, with proven safety and efficacy [8-10]. 
Other local anaesthetics that are used include: amethocaine patch, ethylchloride spray, lidocaine gel, lidocaine gel $10 \%$ + glycerrhetinic acid monohemiphtalate disodium (absorption promoter), lidocaine adrenaline tetracaine (LAT) gel, liquid nitrogen tetracaine adrenaline cocaine (TAC) gel, tetracaine cream.

In addition to the above, of the the most commonly used compounded topical anesthetics is the tripleanesthetic benzocaine/lidocaine/tetracaine cream (BLT gel/cream). When properly formulated the BLT gel can provide effective cutaneous anesthesia as early as 15 minutes after application without occlusion, reaching a maximum effect 30 minutes after application.

\section{Aim of the Study}

The aim of the study was to compare the effectiveness of different types of topical anesthetics most commonly utilized in dermatological procedures. The types of topical anesthesia subject to the study were: EMLA cream, BLT cream, infraorbital nerve blocks with $2 \%$ lidocaine with epinephrine. In several cases patients were additionally premedicated with ibuprofen $400 \mathrm{mg}$ or ketoprofen $100 \mathrm{mg}$ prior to the procedure. The goal of the assessment of the operation of these types of topical anesthetics was to establish the best choice for cosmetic dermatology procedures.

\section{MATERIAL AND METHODS}

The study involved 12 patients with scheduled painful dermatological procedures intended to improve their facial and neck skin. The planned procedures were: fractionated $\mathrm{CO} 2$ laser resurfacing-using ECO2 lutronic laser (6 patients) and micro needling using Dermapen (6 patients). Patients' pain levels during procedures were measured using numeric $0-10$ scale (where 0 - no pain, 10 - most severe pain one can imagine). 6 patients were anesthetized topically with EMLA cream and 6 with BLT cream (compounded in local pharmacy). Additionally 2 out of 6 patients in each group had infraorbital nerve blocks with $2 \%$ lidocaine with epinephrine. $2 \mathrm{ml}$ of the local anesthetic was injected on each side to ensure better anesthesia of the peri oral and lip area. 1 patient in the infraorbital nerve block and 2 patients in the topical cream anesthesia only group were pre medicated with paracetamol $1000 \mathrm{mg}$, ibuprofen $400 \mathrm{mg}$ or ketoprofen $100 \mathrm{mg}$ depending on the patient's preference.

\section{RESULTS}

In order to compare the results of the various types of anesthesia as well as combinations of different forms of anesthesia used, the patient self-report responses were disaggregated into the following 4 groups and 3 subgroups defined by the method of anesthesia applied:

Group 1 (topical EMLA only) - 3 patients

Group 2 (topical BLT only) -3 patients

Group 3 (topical EMLA and infraorbital nerve block) -1 patient

Group 4 (topical BLT and infraorbital nerve block) -2 patients

Subgroup 5 (topical EMLA and premedicationketoprofen 100mg) -l patient

Subgroup 6 (topical BLT and premedication ibuprofen $400 \mathrm{mg}$ ) - - patient

Subgroup 7 (topical EMLA, infraorbital nerve block and ketoprofen $100 \mathrm{mg}$ ) -l patient.

Pain intensity was measured using 0-10 numeric scale (10 being the worst imaginable pain and 0 no pain). Patients were asked to assess their pain levels during fractionated CO2 laser treatment of the face. The patient in subgroup 7 scored the lowest (4) while the results in group 1 (EMLA only) displayed the highest pain scores (8.6). Other groups/subgroups scores ranged from 6 to 9: group 2-(8), 3-(7), 4-(6), 5-(8), 6-(9).

The results of the study indicate that the application of topical anesthesia in the form of cream of gel (in this case the most commonly used EMLA and BLT creams were compared - groups 1 and 2) has only a very moderate anesthetic effect during painful dermocosmetic procedures. This method of anesthesia stands out as the easiest in administration and does not involve any pain at the stage of application. However, as a standalone anesthetic, these creams are not fully effective.

The numbing effect of the EMLA and BLT creams applied in combination with premedication by painkillers - ketoprofen and ibuprofen (groups 5 and 6) was also very limited. The study showed that the premedication by these substances failed to significantly reinforce the numbing effect of the creams alone. The comparison of mean pain level in groups with EMLA and BLT creams only (group 1 and 2) and groups applying the creams and premedicated (groups 5 and 6) are almost the same which questions the rationale behind the use of these drugs. 
In groups 3 and 4 the anesthetic EMLA and BLT creams were applied in combination with an infraorbital nerve block injection. Both groups demonstrated similar pain levels, still considerably high and not significantly lower than the results for standalone application of creams.

The only noticeably lower pain level was associated with the patient in group 7, whose anesthesia consisted of three different methods: the topical application of EMLA, infraorbital nerve block and premedication with ketoprofen $100 \mathrm{mg}$. This triple anesthesia proved to be by far the most effective method from the range of methods assessed in the present study.

\section{DISCUSSION AND CONCLUSIONS}

Finding the ideal topical anesthetic is one of the greatest challenges of present-day cosmetic dermatology. One can assume that the most desired method of anesthesia in this context would have to be easy to apply, display high clinical effectiveness over a short time period, be able to exert numbing effect on intact skin without systemic effects, cause nominal pain or discomfort during treatment with minimal to no side effects [11,12].

The reported study demonstrated that patients subjected to treatment with the use of anesthetic EMLA and BLT creams alone, similarly to those who were previously premedicated with ibuprofen and ketoprofen still feel a considerable level of pain during the performed dermocosmetic procedures. The mean pain level in these groups was also very similar to patients who were anesthetized with the creams and infraorbital nerve block injection. The most effective method of anesthesia was proved to be the triple anesthesia involving of a combination of a painkiller drug, EMLA cream and infraorbital nerve block.

Of course, one has to keep in mind that the choice of which anesthetic to use depends on a number of factors, including personal patient factors, age, pregnancy status, state of health, history of allergies, other medical conditions such as renal or hepatic failure, cardiac problems, current medications, type of procedure being performed, its duration, body area where the procedure will be carried out, doctor's own preference, training and experience and the patient's preference including for example severe needles phobia.

Only after a careful consideration of all these factors and a detailed patient interview, the doctor will be able to make an informed decision about the choice of the best local anesthetic for the particular procedure planned.

\section{Statement of Human and Animal Rights}

All procedures followed were in accordance with the ethical standards of the responsible committee on human experimentation (institutional and national) and with the Helsinki Declaration of 1975, as revised in 2008 .

\section{Statement of Informed Consent}

Informed consent was obtained from all patients for being included in the study.

\section{REFERENCES}

1. Sobanko JF, Miller CJ, Alster TS. Topical anesthetics for dermatologic procedures: a review. Dermatol Surg. 2012;38:709-21.

2. Railan D, Alster TS. Use of topical lidocaine for cosmetic dermatologic procedures. J Drugs Dermatol. 2007;6:1104-8.

3. Alster TS. The lidocaine/tetracaine peel: a novel topical anesthetic for dermatologic procedures in adult patients. Dermatol Surg. 2007;33:1073-81.

4. Cohen JL, Gold MH. Evaluation of the efficacy and safety of a lidocaine and tetracaine $(7 \% / 7 \%)$ cream for induction of local dermal anesthesia for facial soft tissue augmentation with hyaluronic acid. J Clin Aesthet Dermatol. 2014;7:32-7.

5. Chiang YZ, Al-Niaimi F, Madan V. Comparative Efficacy and Patient Preference of Topical Anaesthetics in Dermatological Laser Treatments and Skin Microneedling. J Cutan Aesthet Surg. 2015;8:143-6.

6. Forster R, Pagnamenta F. Dressings and topical agents for arterial leg ulcers, Cochrane Database Syst Rev. 2015;29:CD001836.

7. Vinklárková L, Masteiková R, Vetchý D, Doležel P, Bernatonienė J. Formulation of Novel Layered Sodium Carboxymethylcellulose Film Wound Dressings with Ibuprofen for Alleviating Wound Pain. Biomed Res Int. 2015;2015:892671.

8. Asilian A, Shahmoradi Z, Mazloomi R, Nilforushzadeh MA. The effects and side effects of lidocaine tetracaine peel off on laserassisted hair removal. Adv Biomed Res. 2014;3:110.

9. Kumar M, Chawla R, Goyal M. Topical anesthesia. J Anaesthesiol Clin Pharmacol. 2015;31:450-6.

10. Gaitan S, Markus R. Anesthesia methods in laser resurfacing. Semin Plast Surg. 2012;26:117-24.

11. Alster T. Review of lidocaine/tetracaine cream as a topical anesthetic for dermatologic laser procedures. Pain Ther. 2013;2:11-9.

12. Kim WO, Song BM, Kil HK. Efficacy and safety of a lidocaine/ tetracaine medicated patch or peel for dermatologic procedures: a meta-analysis. Korean J Anesthesiol. 2012;62:435-40.

Copyright by Jacek Zdybski, et al. This is an open-access article distributed under the terms of the Creative Commons Attribution License, which permits unrestricted use, distribution, and reproduction in any

medium, provided the original author and source are credited.

Source of Support: Nil, Conflict of Interest: None declared. 